
Not for reproduction, distribution or commercial use.

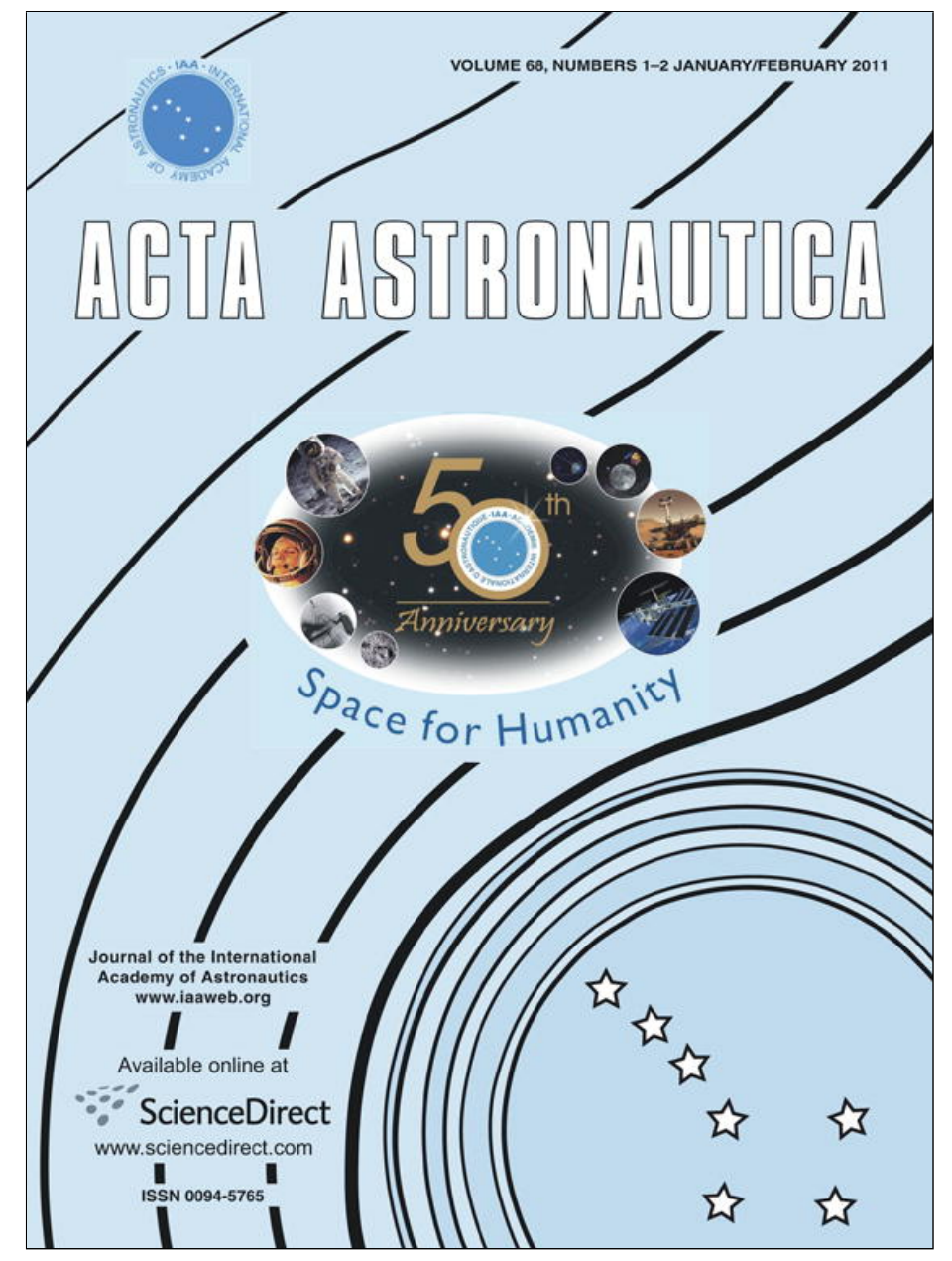

This article appeared in a journal published by Elsevier. The attached copy is furnished to the author for internal non-commercial research and education use, including for instruction at the authors institution and sharing with colleagues.

Other uses, including reproduction and distribution, or selling or licensing copies, or posting to personal, institutional or third party websites are prohibited.

In most cases authors are permitted to post their version of the article (e.g. in Word or Tex form) to their personal website or institutional repository. Authors requiring further information regarding Elsevier's archiving and manuscript policies are encouraged to visit:

http://www.elsevier.com/copyright 


\title{
Analysis of predictive entry guidance for a Mars lander under high model uncertainties
}

\author{
Alexander I. Kozynchenko \\ Finnstastigen 64, SE-197 35 Bro, Stockholm, Sweden
}

\section{A R T I C L E I N F O}

\section{Article history:}

Received 20 May 2010

Received in revised form

1 August 2010

Accepted 11 August 2010

Available online 30 August 2010

\section{Keywords:}

Mars lander

Entry phase

Predictive guidance

Precision landing

Atmospheric uncertainties

Adaptation

\begin{abstract}
A B S T R A C T
The problem of precision landing on Mars is now considered to be an essential challenge in the planned Mars missions. The paper focused on the guided atmospheric entry as a predominant phase in achieving a desired target state, as compared with the following parachute and powered descent. The predictive algorithms for the longitudinal guidance of a low-lift entry vehicle are treated. The purpose is to investigate applicability of the predictive strategy under possible high discrepancies between the on-board dynamic model and real environment while in entry trajectory. The comparative performance analysis based on computer simulation has been made between the standard one-parametric "shooting" predictive algorithm and a more complex two-parametric algorithm providing lower final velocity and, thus, expanding the interval of admissible downrange. However, both algorithms display considerable degradation of downrange accuracy in the cases when the actual drag force is larger than the modelled one. An acceptable solution has been found by including to both predictive guidance schemes an identification algorithm that repeatedly adapts the on-board model to varied environment in real time scale.
\end{abstract}

(c) 2010 Elsevier Ltd. All rights reserved.

\section{Introduction}

The paper is concerned with the problem of precision landing of a capsule-type spacecraft on the Martian surface. Till date, all Mars landers having reached the surface successfully - Mars 3, Viking 1 and 2, Mars Pathfinder, Mars Exploration Rovers (A and B), Phoenix - had been targeted to landing sites only by approach navigation before atmospheric entry, having resulted in large dispersion ellipses with major axis lengths on the order of $100 \mathrm{~km}$ (see, e.g., [1,2]). These spacecrafts had not been equipped with a closed-loop guidance system operating at the entry phase, which plays the main role in achieving a desired landing accuracy within the so-called EDL sequence, i.e., the hypersonic ballistic or lifting entry, parachute descent, and powered landing. However, despite the absence of actual guidance systems on board of any Mars landers so far,

E-mail address: alexander_kozynchenko@yahoo.se considerable efforts had been made since 1970s in developing guidance algorithms in entry to the Martian atmosphere. Initially, the research had been focused on a vital condition of achieving the final velocity as minimal as possible till the moment of parachute deployment (or, equivalently, finding the maximal final altitude), whereas the precision landing requirements are considered to be less essential [3]. Such a choice was caused by the difficulties to satisfy soft landing conditions after the entry phase, since a re-entry vehicle has to be designed with a very large ballistic coefficient $\sigma=0.5 C_{D} S \mathrm{~m}^{-1}$ in order to slow down properly in highly rarefied Martian atmosphere with large, hardly predictable variations. Research efforts in the last decade continue to be concentrated on solving the problem of maximizing the final altitude at entry phase [4,5] in view of the planned complex unmanned Mars missions like Mars Science Laboratory (MSL) [6] as well as eventual human explorations with new EDL challenges discussed in $[7,8]$. At the same period, some articles have appeared $[1,2,9-11]$, which are centred on the development of more 


$\begin{array}{ll}\text { Nomenclature } \\ \text { Acronyms } \\ \text { AoA } & \text { angle of attack } \\ \text { BVP } & \text { boundary-value problem } \\ \text { EDL } & \text { entry, descent, and landing } \\ \text { INS } & \text { inertial navigation system } \\ \text { IVP } & \text { initial-value problem } \\ \text { MSL } & \text { Mars Science Laboratory } \\ & \\ \text { Variables } & \text { and parameters } \\ & \\ C_{A} & \text { axial force coefficient } \\ C_{N} & \text { normal force coefficient } \\ C_{D} & \text { drag coefficient } \\ C_{L} & \text { lift coefficient } \\ D & \text { drag force, } \mathrm{N} \\ d T & \text { interval between control corrections, } \mathrm{s} \\ d t & \text { time step of numerical integration, } \mathrm{s} \\ \Delta x_{f} & \text { downrange miss, m } \\ g & \text { local gravitational acceleration on Mars, } \mathrm{m} / \mathrm{s}^{2} \\ H & \text { vehicle altitude above the Martian reference } \\ K & \text { sphere, m } \\ L & \text { coefficient of model discrepancy } \\ L / D & \text { lift force, } \mathrm{N} \\ M & \text { lift-to-drag ratio } \\ M & \text { vehicle mass, kg } \\ & \text { Mach number }\end{array}$

\begin{tabular}{|c|c|}
\hline$N$ & axial load factor \\
\hline$Q$ & dynamic pressure, $\mathrm{Pa}$ \\
\hline$R$ & radius of Mars, $\mathrm{m}$ \\
\hline$S$ & aeroshell reference area, $\mathrm{m}^{2}$ \\
\hline$t$ & time, $\mathrm{s}$ \\
\hline$V$ & $\begin{array}{l}\text { vehicle velocity relative to the Martian surface, } \\
\mathrm{m} / \mathrm{s}\end{array}$ \\
\hline$x$ & $\begin{array}{l}\text { downrange, longitudinal range over the } \\
\text { Martian spherical surface, } m\end{array}$ \\
\hline$\alpha$ & angle of attack, rad \\
\hline$\delta x$ & $\begin{array}{l}\text { admissible downrange error in guidance } \\
\text { algorithms, } \mathrm{m}\end{array}$ \\
\hline$\theta$ & flight-path angle, rad \\
\hline$\xi$ & $\begin{array}{l}\text { angle of slope of the line representing a } \\
\text { 2-parametric control function, rad }\end{array}$ \\
\hline$\rho$ & atmospheric density, $\mathrm{kg} / \mathrm{m}^{3}$ \\
\hline & ballistic coefficient, $\mathrm{m}^{2} / \mathrm{kg}$ \\
\hline
\end{tabular}

\section{Subscripts}

0 initial value; value at the Martian spherical surface

$f \quad$ value of a variable at termination of entry phase

$j \quad$ value of a variable at $j$ th control correction

$m \quad$ value of a variable in on-board model

min, mid, $\max$ minimal, middle, and maximal values

$t_{f} \quad$ target value at termination of entry phase precise closed-loop entry guidance systems with a target miss less than $10 \mathrm{~km}$ at the parachute deployment height. Mease et al. [2] suggested the guidance algorithm based on the concept of planning and tracking drag similar to that used by the space shuttle. This work continued earlier investigations done by $\mathrm{Tu}$ et al. [9], where a closed-loop entry guidance law was developed for low-lift Mars landers (having lift-to-drag ratio of $0.06 \leq L / D \leq 0.18$ ). Carman et al. [10] and then Mendeck and Carman [11] presented a reference-path entry guidance method using the guidance technique of Apollo second entry phase adapted for Mars landing conditions. Powell [12] investigated the performance of the numerical predictor-corrector technique to guide a Mars lander to both the aerocapture objective and precision landing one, namely, to deploy a parachute within $10 \mathrm{~km}$ of a target. A more complex predictor-corrector technique having decoupled angle-of-attack (AoA) and bank angle control was also considered by Jits and Walberg [13] for the aerocapture case with the focus on guidance under multiple off-nominal conditions, including significant atmospheric and aerodynamic dispersions. Kluever [1] carried out the comparative performance analysis of pre-planned reference-path and predictive path-planning entry guidance methods for a lander modelled on the Mars Science Laboratory mission. In both the cases the bank angle reversals are used to modulate the lift force for longitudinal and lateral trajectory control. Having conducted an extensive 500-run Monte Carlo study, Kluever came to conclusion that the reference-path guidance is likely the better option for a Mars precision landing than the predictive guidance. This opinion seemed to be rested on the main drawback of predictive guidance, namely, its inherent dependence on the adequacy of on-board model of a lander entry motion. It should be noted, however, that this conclusion may not be considered as definitive until more comprehensive investigations of this issue would be made. There are a number of predictive guidance methods, which, from the mathematical point of view, solve the corresponding eigenvalue problem for ordinary differential equations [14]. This is, for example, the "predictor-corrector" algorithm widely used in planet entry guidance and being, in essence, the "shooting" method applied for solving boundary-value problems (BVP) and differential eigenproblems. Here, eigenvalues are controls ( $L / D$ commands), and eigenfunctions are solutions, i.e., time dependencies of velocity, downrange, etc. In this sense, Kluever's predictive guidance method may be treated as a heuristic kind of alternative so-called expansion methods of solving BVP and differential eigenproblems described in [15].

This paper provides further analysis of the applicability of predictive guidance technique at the entry phase with the focus on functioning under high atmospheric and aerodynamic uncertainties and shows the accuracy limitations inherent to the investigated predictive algorithms. The emphasis is placed on longitudinal guidance that is considered to be a more challenging 
problem for the given case in comparison with a crossrange guidance. Again, the paper discusses possible measures to enhance the terminal downrange accuracy at the entry phase and describes an adaptive modification of guidance algorithms.

\section{Longitudinal model of lifting entry of a Mars lander}

At the current preliminary stage of comparative analysis of different guidance algorithms, when only most essential issues are to be detected, it would be worthwhile using a relatively simple mathematical model of Mars lander motion. In the paper a simplified model is considered which includes a point-mass vehicle with a low lift-to-drag ratio making unpowered guided descent in a vertical plane over a spherical, non-rotating planet in the motionless Martian atmosphere. In the model description the following Cartesian coordinate systems are used (see Fig. 1) - topocentric fixed initial frame $O_{e} x_{n e} y_{n e}$ locating the entry point $E$ of a vehicle, vehiclecentred local frame $O x_{n} y_{n}$, body-fixed frame $O x_{1} y_{1}$, velocity frame $O x y,[16]$.

The set of four first-order ordinary differential equations consists of two force equations in terms of two state variables $(V, \theta)$ in the velocity frame, as well as two kinematic equations in terms of the trajectory coordinates $(h, x)$ defined in a Mars-centred polar frame, the polar axis of which coincides with the positive $O_{e} y_{n e}$-axis

$\dot{V}=-\frac{D}{m}-g \sin \theta$,

$\dot{\theta}=\frac{L}{m V}-\left(\frac{g}{V}-\frac{V}{R+h}\right) \cos \theta$,

$\dot{h}=V \sin \theta$,

$\dot{x}=\frac{V R}{R+h} \cos \theta$,

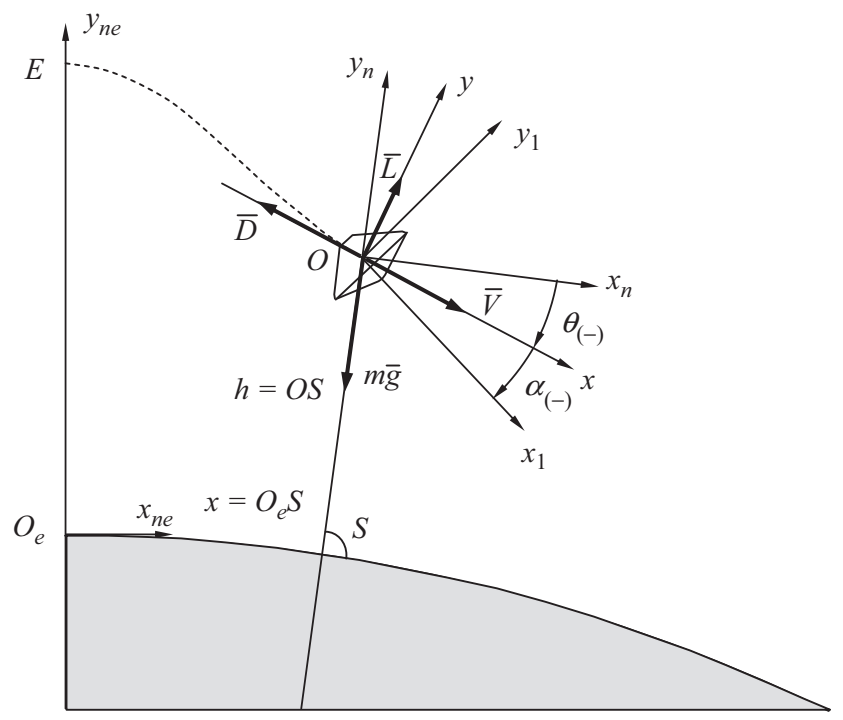

Fig. 1. Coordinate systems, angles, and forces being considered at the entry phase of Mars lander mission. where drag and lift forces are determined by the AoA $\alpha$ taken as a control variable, as well as by axial and normal force coefficients, and dynamic pressure

$D=C_{D} S q$,

$L=C_{L} S q$,

$C_{D}=C_{N} \sin \alpha+C_{A} \cos \alpha$,

$C_{L}=C_{N} \cos \alpha-C_{A} \sin \alpha$,

$q=\frac{1}{2} \rho V^{2}$,

with gravitational acceleration calculated by Newton's inverse-square force law

$g=g_{0}\left(\frac{R}{R+h}\right)^{2}$.

Atmospheric density $\rho$ is computed on the base of the atmospheric model 40NO presented in [17] (p. 12, Table 6.5) and providing a density altitude profile for $35-50^{\circ}$ latitudes in the northern hemisphere. An approximate profile used in the paper and shown in Fig. 2 has been created as a piecewise continuous function consisting of exponential laws between reference points A, B, C, and D at altitudes of $130,40,20$, and $0 \mathrm{~km}$. These laws are as follows:

$$
\begin{aligned}
& \mathrm{AB}: \rho=3.7 \times 10^{-4} \exp \left(-1.371 \times 10^{-4}\left(h-40 \times 10^{3}\right)\right), \\
& \mathrm{BC}: \rho=2.8 \times 10^{-3} \exp \left(-1.012 \times 10^{-4}\left(h-20 \times 10^{3}\right)\right), \\
& \mathrm{CD}: \rho=0.01417 \exp \left(-0.8109 \times 10^{-4} h\right) .
\end{aligned}
$$

Parameters of the motion model (1)-(4) are chosen for the case study considered in this paper, which involves a Mars lander model similar to that of the planned MSL mission. The vehicle has an entry mass $m=2200 \mathrm{~kg}$ and a sphere-cone aeroshell with high semiapex angle, diameter $4.0 \mathrm{~m}$, and a reference area $S=12.57 \mathrm{~m}^{2}$. The permissible range of changing the $A \circ A$ is taken to be $\alpha \in\left[-15^{\circ}, 15^{\circ}\right.$. In this range and at hypersonic velocity, the shape has a high

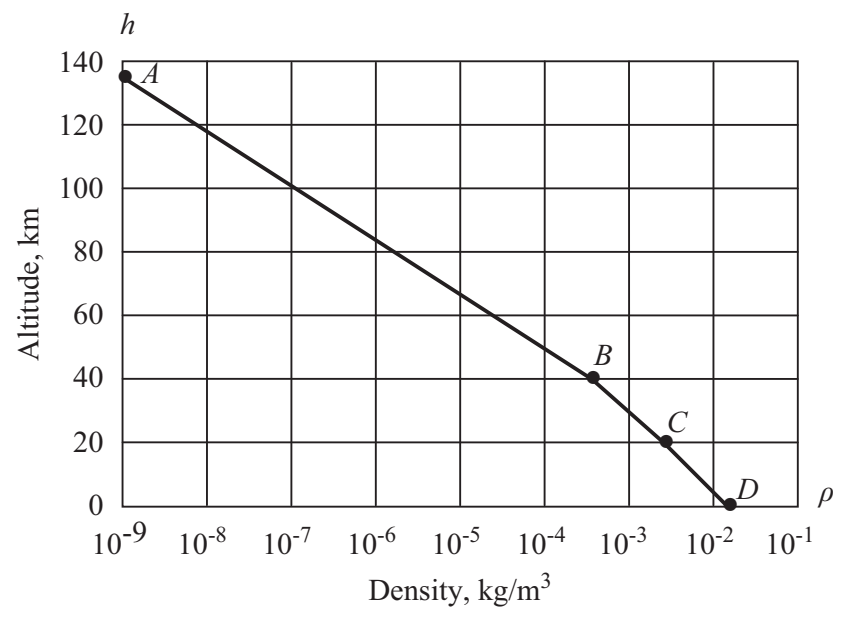

Fig. 2. Density profile for Martian atmosphere assumed for this case study (approximation of the model 40NO [17]). 
axial force coefficient $C_{A}=1.60$, very small normal force coefficient $C_{N}$ being taken to be zero, and a low lift-to-drag ratios $L / D<0.27$. The gravitational acceleration at the Martian reference sphere of radius $R=3395 \mathrm{~km}$ is $g_{0}=3.716 \mathrm{~m} / \mathrm{s}^{2}$.

\section{Description of the entry guidance problem}

\subsection{Mathematical formulation}

A guidance problem at the entry phase for the Mars lander model described in the previous section consists in finding the AoA as a restricted function of time $\alpha(t), \alpha_{\min } \leq \alpha(t) \leq \alpha_{\max }$. This function is calculated on-board first at the beginning of entry $t_{0}=0$ and it is defined up to some unspecified final time $t_{f}$, when the target point would be achieved. An inertial navigation system (INS) provides values of state variables at the entry point $E$ (Fig. 1), forming a set of initial conditions

$V\left(t_{0}\right)=V_{0}, \quad \theta\left(t_{0}\right)=\theta_{0}, \quad h\left(t_{0}\right)=h_{0}, \quad x\left(t_{0}\right)=x_{0}$.

The solution must meet the following terminal condition:

$h_{f} \equiv h\left(x=x_{t_{f}}\right)=h_{t_{f}}$

and the terminal velocity constraint

$V_{f} \equiv V\left(h=h_{t_{f}}\right) \leq V_{t_{f}}$

which correspond to reaching the designated target downrange $x_{t_{f}}$ at required parachute-deployment target state $\left(h_{t_{\mathrm{f}}}, V_{t_{f}}\right)$. Other constraints and optimal criteria (e.g., load factor, maximal temperature) being important and even crucial in the case of entry in thicker atmospheres like Earth's, are not taken into account in the current analysis.

As has been pointed out by Kozynchenko[18-20], the problems such as those described can be classified as generalized BVPs or, more specifically, as a differential eigenvalue problem already mentioned in Section 1. A strong formulation of this problem for a general case was given in [21]: differential eigenproblems are BVPs for a system of $p$ ordinary differential equations of the first order

$\overrightarrow{\dot{x}}=\vec{f}(t, \vec{x} ; \vec{\lambda})$,

where $\vec{f}=\left\{f_{1}, \ldots, f_{p}\right\}$ is the $p$-vector of right-hand sides; $\vec{\lambda}=\left\{\lambda_{1}, \ldots, \lambda_{q}\right\}$ the $q$-vector of sought-for parameterseigenvalues; $\vec{x}=\left\{x_{1}, \ldots, x_{p}\right\}$ the $p$-vector of sought-for functions - eigenfunctions; the number of boundary conditions equals to $p+q$.

In this case, the number of initial conditions (12) equals to $p=4$. In the paper, we will analyse first a simplest, one-parametric predictive guidance with only one control variable, namely, an AoA $\alpha=$ const. That is, only one terminal condition-(13) or (14) — may be taken into account. The fulfilment of velocity condition (14) is crucial, otherwise the soft landing is impossible. If the terminal condition (13) of landing a vehicle at the pre-selected site will not be satisfied, the vehicle still would have the possibility of soft landing, though not guaranteed due to eventual surface hazards (craters, trenches, etc.) and relief elevation uncertainties. However, in order to solve the given eigenvalue problem we choose the condition of precision landing (13), checking the accomplishment of the constraint (14) on a solution and discarding the latter if final velocities were too high.

In the paper, predictive guidance technique is analysed on the base of an object-oriented simulation model that includes a class describing the real motion of a Mars lander, as well as classes involving the on-board environment and motion models, and the predictive guidance algorithm. In the current investigation, the navigation errors are not considered in the simulation model, so a class of on-board inertial navigation system is omitted.

Before studying and simulation of the guidance problems, a simulation study of general features of the entry-phase trajectories for different AoAs $\alpha_{\min } \leq \alpha \leq \alpha_{\max }$ taken as a constant over a trajectory has been performed. The following initial conditions for the systems (1)-(4) are taken: $V_{0}=6000 \mathrm{~m} / \mathrm{s}, \theta_{0}=-15^{\circ}, h_{0}=125 \mathrm{~km}, x_{0}=0 \mathrm{~km}$. The numerical integration of the system is accomplished by a standard 4th-order Runge-Kutta method with a fixed time step $d t=0.01 \mathrm{~s}$. The trajectory integration is terminated when the target altitude $h_{t_{f}}=7 \mathrm{~km}$ is reached, the terminal velocity $V_{f}$ being compared with the constraint $V_{t_{f}}=400 \mathrm{~m} / \mathrm{s}$. Dynamic pressure and Mach number at parachute deployment under these terminal conditions are $q \approx 620 \mathrm{~N} / \mathrm{m}^{2}$ and $M \approx 1.65$, respectively, i.e. less than those for the MSL mission-750 N/m $\mathrm{m}^{2}$ and 2.0, respectively (see [7], Table 1). Given boundary values are close to those used in [1]. All simulation models presented in the paper have been developed using the Microsoft Visual $\mathrm{C}++2008$ Express Edition platform. Fig. 3(a) shows an attainable trajectory bunch bounded by paths with extreme values of AoA, so that the limits of the range attainability domain are equal to $x_{t_{f} \text { min }}=469 \mathrm{~km}$ and $x_{t_{f} \text { max }}=935 \mathrm{~km}$. Fig. 3 (b) presents the terminal velocity $V_{f}$ vs. final downrange $x_{f} \in\left[x_{t_{f} \text { min }}, x_{t_{f} \text { max }}\right]$. As can be seen, the terminal velocity constraint (14) is satisfied for the given case study at only ca. $48 \%$ of the aforementioned range of attainability; thus reducing the trajectory bunch up to the new limits $x_{f C}=586 \mathrm{~km}$ and $x_{f D}=811 \mathrm{~km}$, and forming a domain of admissible paths. Admittedly, at the right interval $D B$ the function $V_{f}\left(x_{f}\right)$ is near to the constant value $V_{f} \approx 405 \mathrm{~m} / \mathrm{s}$, which exceeds the given target value $V_{t_{f}}=400 \mathrm{~m} / \mathrm{s}$ by less than $2 \%$.Therefore, the domain of admissible paths can be safely extended up to the maximal downrange (point $B$ ).

Finally, the maximum axial load factor $n_{\max }=D_{\max } / \mathrm{mg}$ should be mentioned, which, being computed over feasible trajectories of the ensemble, decreases monotonously from its maximal value $n_{\max }=17.9$ for the path with AoA $\alpha_{\max }=15^{\circ}$ to $n_{\max }=10.0$ for the path with $\alpha_{\min }=-15^{\circ}$.

\subsection{Navigation aspects of the guidance at entry phase}

Although the principal aim of the current research is to study the performance of the proposed algorithms as such, without regard to navigation errors, it is worthwhile to 
a

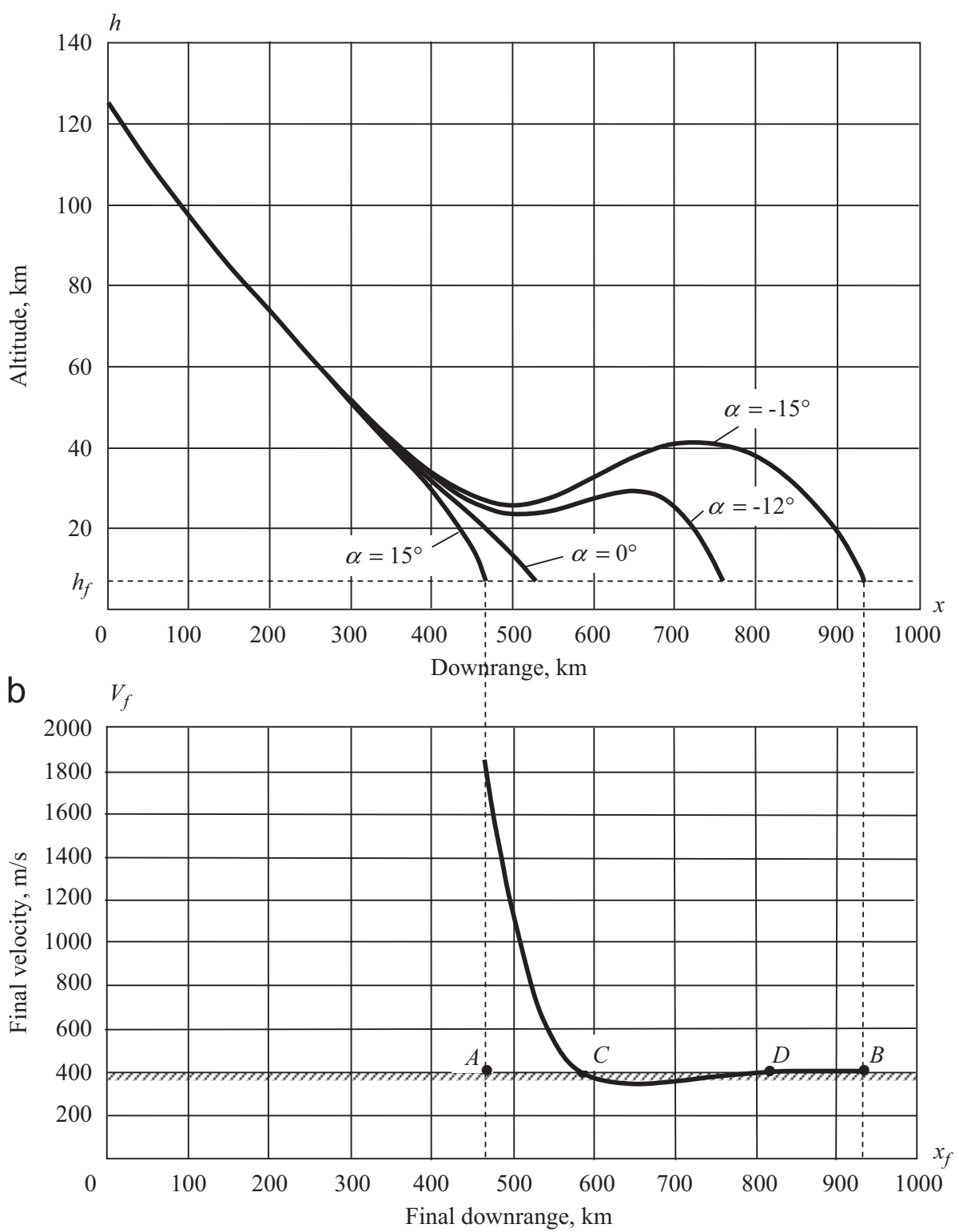

Fig. 3. A bunch of descent trajectories of a Mars lander within attainability domain (a) and the corresponding dependence of final velocity on the final downrange (b).

describe the primary problem of precision landing on Mars more broadly with mentioning the navigation aspects of a mission. The predictive guidance algorithms need to get complete information about the current state vector of the lander (i.e., velocity and position) both at the beginning of the entry phase and at each control corrections over the path. This task is accomplished by an onboard astro-inertial navigation system being analogous to that to be used in MSL [6], which includes a star scanner for determining the lander attitude during cruise, and a strapdown inertial measurement unit equipped with three accelerometers and three laser gyroscopes. Approach navigation is performed using radiometric tracking data provided by the Deep Space Network. Before separating the cruise stage, the available navigation data (position, velocity, and attitude) are uploaded to on-board computer that accomplishes initialization of the INS and starts processing the data incoming from motion and rotation sensors (i.e., noise filtering, calculating angles of orientation, and integrating the navigation equations for determining the current velocity vector and position of a lander).

However, despite the measures on improving the initialization accuracy during approach navigation, there still exists a risk of arising marked errors in initial position and orientation of velocity vector. Obviously, these errors will rapidly accumulate with time if the INS is applied alone. The predictive algorithms discussed in the paper are able to compensate considerable initialization errors, but only on condition that the subsequent incoming navigation information at entry phase would be more accurate. It can be done by integrating the INS with an auxiliary navigation subsystem and forming, e.g., such combined system as a visual-aided INS (see, e.g. [22]), which provides the landmark-based estimation of position and velocity. In this case, 
the extended Kalman filter is proposed to be applied to fuse inertial measurements with camera observations of the socalled map landmarks, i.e., features (e.g., craters) whose coordinates can be found from a map of the landing site, which is available a priori.

\section{Predictive guidance using conventional one-parametric path-planning "shooting" algorithm}

We will start the investigation of predictive entry guidance with the commonly used one-parametric "shooting" algorithm. At the entry point of a trajectory, the inertial navigation system passes information about current state variables of the lander to the on-board computer which, taking this data as initial conditions, solves a one-parametric eigenvalue problem and computes an output - the control variable profile $\alpha(x)$ to be constant in this simple case up to reaching the target values of downrange $x_{t_{f}}$ or altitude $h_{t_{f}}$. The final velocity $V_{f}$ is compared with the target velocity $V_{t_{f}}$ and, if $V_{f} \leq V_{t_{f}}$, the given terminal guidance problem is considered to be feasible. That is, initial conditions at the entry point

a

Interval between control corrections $d T=5 \mathrm{sec}$
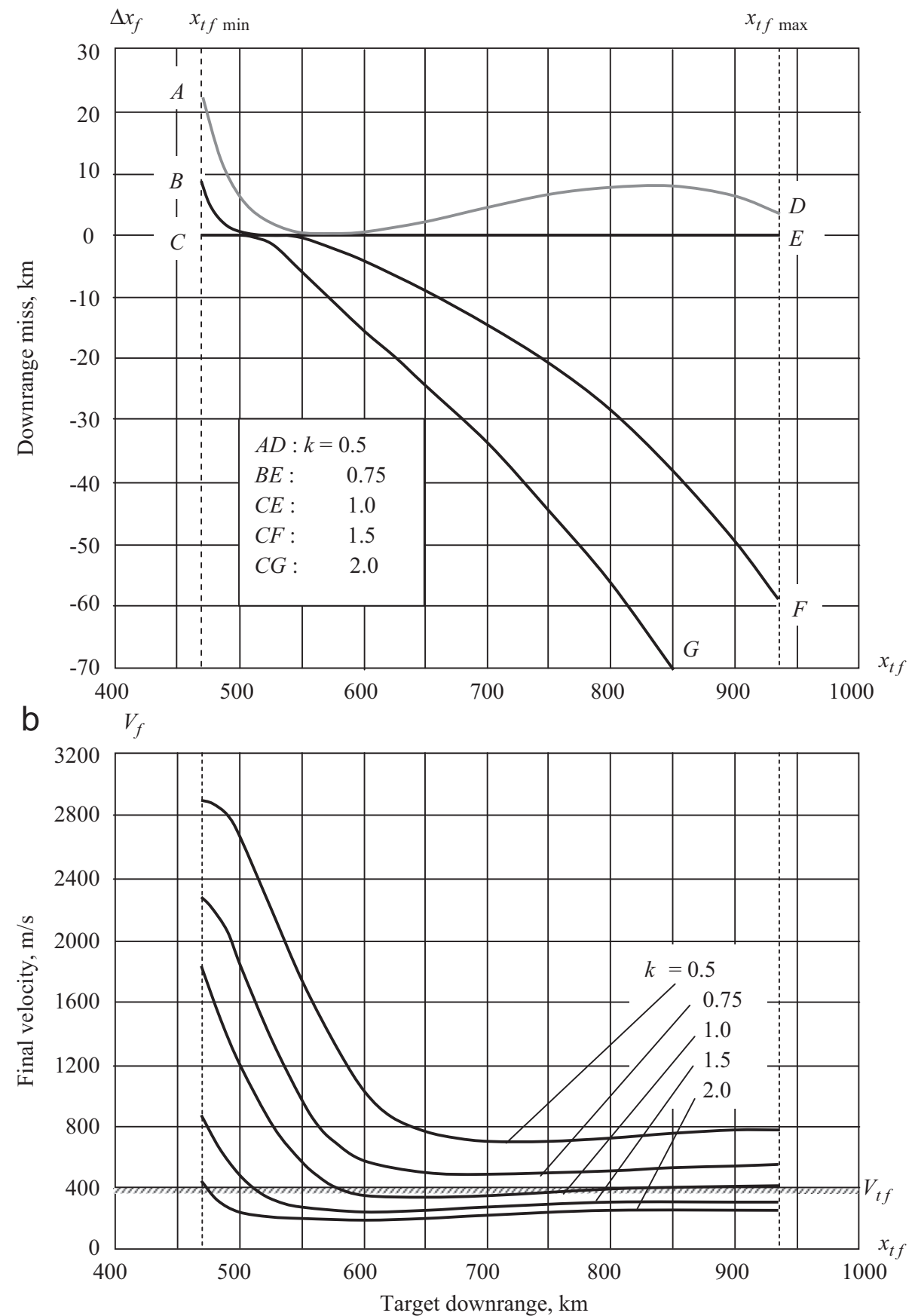

Fig. 4. Classic one-parametric predictive guidance: dependences of downrange miss $\Delta x_{f}$ (a) and final velocity $V_{f}$ (b) on target downrange $x_{t_{f}}$ and coefficient of model discrepancy $k$. 
provided by the approach navigation must be chosen in such a way that the target downrange $x_{f}$ will lie within the domain of admissible paths, i.e. $\left[x_{C}, x_{B} \equiv x_{f}\right]$ in the case study being considered.

The "shooting" algorithm studied in the paper starts with bracketing the root $\alpha$ of a non-linear equation

$\Delta x_{f} \equiv x_{f}-x_{t_{f}}=f(\alpha), \quad \alpha \in\left[\alpha_{\min }, \alpha_{\max }\right]$,

and then finding the root with desired degree of accuracy $\delta x_{f}$, using the bisection method. Each ith iteration in the "shooting" algorithm represents a numerical integration of the system of differential Eqs. (1)-(4) with initial conditions (12) and AoA $\alpha_{i}$ corresponding to a midpoint of the current bracketing interval. In other words, at each iteration (bisection) we solve a 4th-order initial value problem, or IVP. Being applied at the entry point $E$ of a trajectory (Fig. 1), the algorithm finds a solution after 6-14 iterations (depending on a target downrange $x_{t_{f}}$ ) for the given case study conditions and $\delta x_{f}= \pm 100 \mathrm{~m}$.

Results of simulation are shown in Fig. 4(a and b). The simulation has been carried out for different target downrange $x_{t_{f}}$ varied within the attainability domain: $x_{t_{f}}$ $\in\left[x_{t_{f \text { min }}}, x_{t_{f \max }}\right]$. Again, particular emphasis has been placed on assessing the guidance performance in the presence of considerable atmospheric dispersions being introduced to the simulation model by a so-called coefficient of model discrepancy $k$ defined as a constant ratio of actual and model aerodynamic forces, i.e.

$k=\frac{L}{L_{m}}=\frac{D}{D_{m}}=$ const.

A guidance scheme involves control corrections over the path every $d T=5 \mathrm{~s}$, when the guidance system solves an eigenvalue problem at new initial conditions and updates the AoA in order to compensate possible atmospheric and aerodynamic disturbances. The value of an interval between control corrections, or update time, $d T$, is chosen on the basis of computer simulation of the guidance process within the range $1.5 \leq d T \leq 10 \mathrm{~s}$ for different values of the coefficient $k \in[0.5,2.0]$. The results show rather weak influence of $d T$ values on both the downrange miss $\Delta x_{f}$ and the final velocity $V_{f}$ within the range. Actually, the interval $d T=5 \mathrm{~s}$ has been taken to be greater enough than the estimated run time needed for guidance data processing (which may take several seconds, see Section 6), actuators and other system delays. Similarly, in the study of predictor-corrector guidance performed in [12], the 5 and $10 \mathrm{~s}$ update times were used.

As we can see in Fig. 4(b), the "shooting" algorithm is not able to decelerate a lander to the desired target velocity $V_{t_{f}}$ within the all attainability domain if actual lift and drag aerodynamic forces are less than or equal to at least $75 \%$ of corresponding model values. Most likely, such a situation may take place for more rarefied atmosphere than its model analogue kept in the on-board computer. On the other hand, the entry guidance with "shooting" algorithm in denser atmosphere $(k>1)$ gives rise to unacceptable downrange miss $\Delta x_{f}$ (falling short) for trajectories with large target downrange, as Fig. 4(a) shows. For example, for $k=1.5$ a span of admissible paths with downrange miss $\Delta x_{f} \leq 10 \mathrm{~km}$ becomes rather short, ca. $150 \mathrm{~km}$, being restricted within the limits $x_{t_{f}} \in[510,660] \mathrm{km}$, and thus placing heavy demands on the accuracy of approach navigation at the entry point.

Therefore, being applied to entry guidance of Mars lander, the conventional "shooting" algorithm is not able to satisfy the terminal conditions, both in target range and velocity, under significant deviations in environment and vehicle parameters, even if its control solution is periodically corrected over the path.

\section{Adapting the entry model of Mars lander in the "shooting" guidance algorithm}

To overcome the drawbacks of the "shooting" algorithm in the presence of considerable model discrepancies, an algorithm of adaptation of the on-board model is suggested in this section. The algorithm represents an on-line identification procedure for a coefficient $k_{m}$-the estimation of the coefficient of model discrepancy $k$ that is considered as an eigenvalue to be found by solving the corresponding differential eigenproblem. As in the case of "shooting" guidance algorithm, we assume that the models of actual and simulated motion of a lander are described by the same set of differential Eqs. (1)-(4). The algorithm consists of the following steps to be done at each control correction:

1. At $j$ th control correction, the current values of state variables $V_{j}, \theta_{j}, h_{j}, x_{j}$ are computed on-board on the data supplied by an inertial navigation system, and then are memorized in order to be used later as initial values in the eigenvalue problem.

2. At the next, $j+1$ st control correction, the actual velocity $V_{j+1}$ is fixed to be used as a target value in the eigenvalue problem.

3. Solving the eigenvalue problem over a time $t_{m} \in\left[t_{j}\right.$, $\left.t_{j}+d T\right]$ by analogy with the guidance problem in Section 4, i.e., starting with bracketing the coefficient $k_{m}$ and then using the bisection to find a root of a non-linear equation

$\Delta V_{j+1} \equiv V_{(j+1) m}-V_{j+1}=\phi\left(k_{m}\right), \quad k_{m} \in\left[k_{m \min }, k_{m} \max \right]$

The estimated coefficient $k_{m}$ is used in the dynamic Eqs. (1) and (2) of the on-board motion model at the $j+1$ st control correction. For example, in this case the adapted Eq. (1) takes the form

$\dot{V}_{m}=-\frac{k_{m}}{2 m_{m}} C_{D m} S_{m} \rho\left(h_{m}\right) V_{m}^{2}-g\left(h_{m}\right) \sin \theta_{m}$

Computer simulation shows that the adaptation algorithm affects the downrange miss $\Delta x_{f}$ considerably, making it negligibly small within the wide downrange span (see Fig. 5(a)). Also, as we can see in Fig. 5(b), the adaptation lowers somewhat the final velocity $V_{f}$. However, the problem of achieving the admissible final velocity $V_{f} \leq V_{t_{f}}$ under descent in more rarefied atmosphere than nominal $(k<1)$ remains the challenging one. 
a Interval between control corrections $d T=5 \mathrm{sec}$

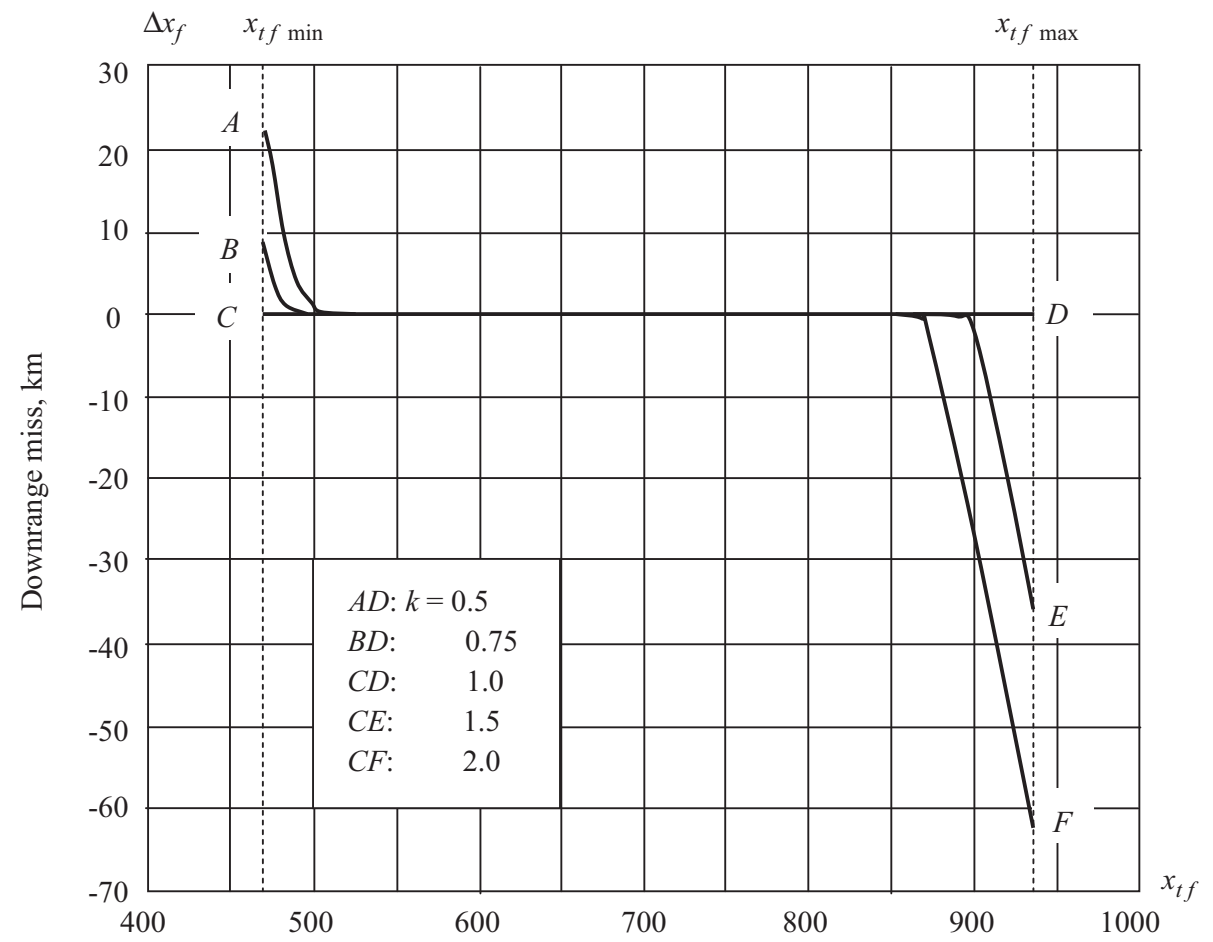

b

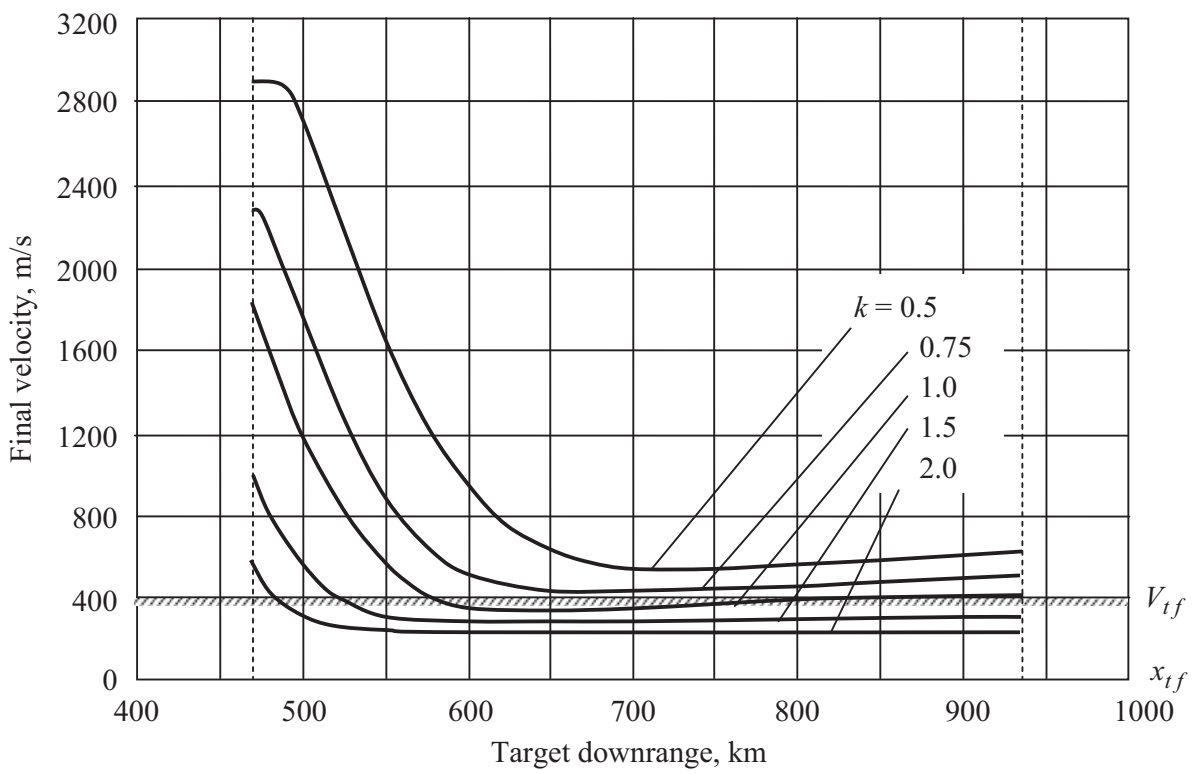

Fig. 5. Classic one-parametric predictive guidance with model adaptation: dependences of downrange miss $\Delta x_{f}\left(\right.$ a) and final velocity $V_{f}$ (b) on target downrange $x_{t_{f}}$ and coefficient of model discrepancy $k$.

\section{Two-parametric planning predictive algorithm and its adaptive version}

From the mathematical point of view, a solution to the guidance problem formulated in Section 3 should be sought in a class of two-parametric control functions, because the corresponding eigenvalue problem involving four initial conditions (12) and two terminal ones (13) and (14) must have two eigenvalues to be found. The twoparametric control function may be chosen from various classes (algebraic, transcendental), but we will take the function being linear within a range $\alpha \in\left[\alpha_{\min }, \alpha_{\max }\right]$, i.e.

$$
\alpha_{j}(x)= \begin{cases}\alpha_{\max }, & \text { if } \alpha_{j}=a_{j}+b_{j}\left(x-x_{j}\right)>\alpha_{\max } \\ a_{j}+b_{j}\left(x-x_{j}\right), & \text { if } \alpha_{\min } \leq \alpha \leq \alpha_{\max } \\ \alpha_{\min }, & \text { if } \alpha_{j}<\alpha_{\min }\end{cases}
$$

where coefficients $a_{j}$ and $b_{j}$ are considered as eigenvalues to be found at each $j$ th step of control correction. Fig. 6(a) shows the graph of the control function (20), where the straight line defined by the linear function encloses the slope angle $\xi$ with the $x$-axis given by $b=\tan (\xi)$. 

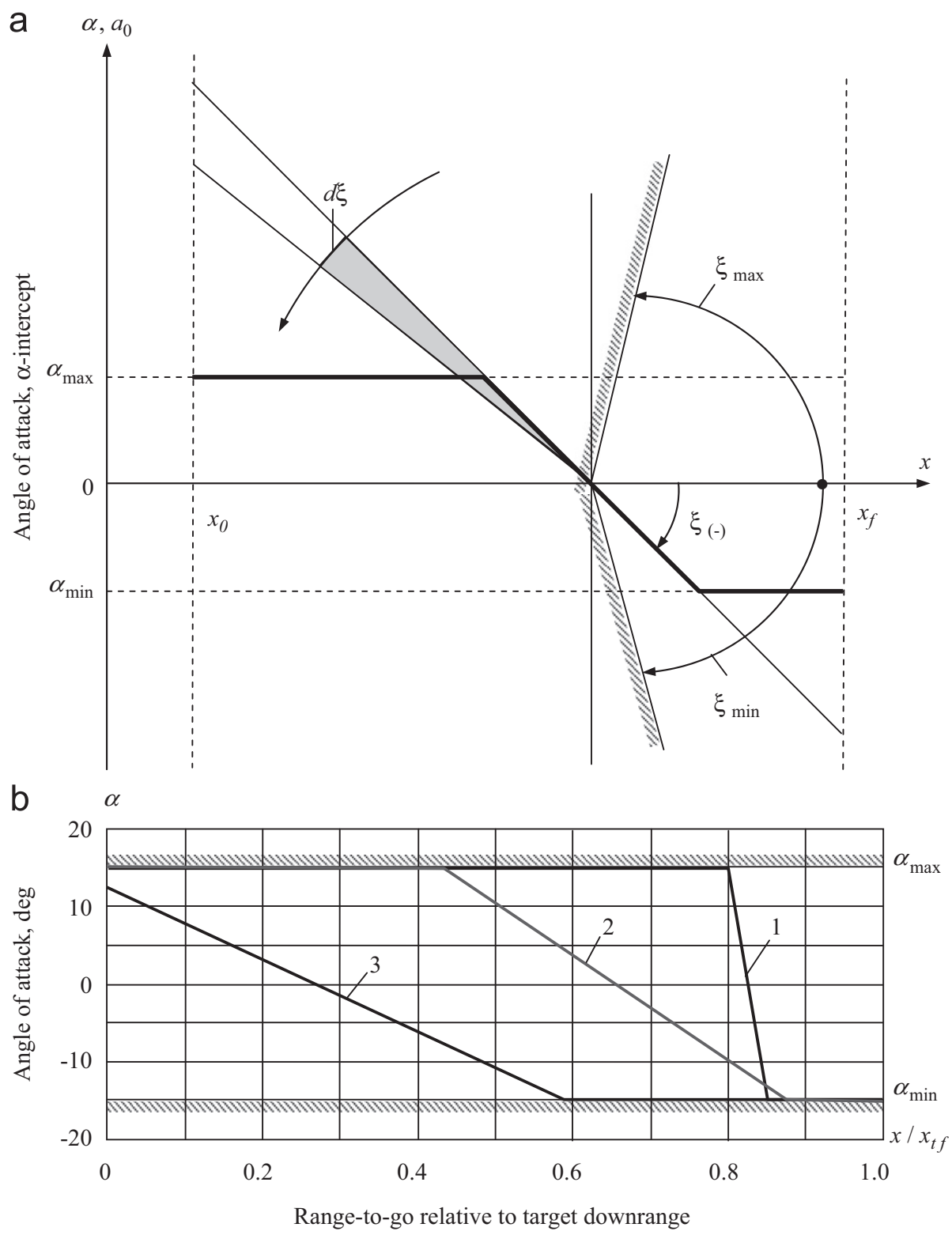

Fig. 6. Two-parametric predictive entry guidance: (a) graphic illustration of constructing the control profile $\alpha(x)$ over the downrange. (b) Control profiles

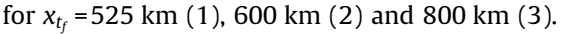

An algorithm for finding the coefficients of control function consists of the following main steps:

(1) The slope angle is set to be zero $\xi_{j}=0$.

(2) To find the single coefficient-intercept $a_{j}$, the one-parametric eigenvalue problem is solved by the "shooting" method similarly to that described in Section 4. The step forms an inner loop of the algorithm. The final velocity $V_{f}$ obtained is fixed.

(3) The slope angle is incremented by a small angle $d \xi$, and the step 2 is repeated to find new values of $a_{j}$ and $V_{f}$.

(4) If the new $V_{f}$ proved to be greater than the original value, it means that the wrong search direction has been chosen, so backtracking and starting search in the opposite direction are fulfilled: the slope angle is decremented by $2 d \xi$, and step 2 is repeated.

(5) After detecting a right direction of searching a minimum value of the final velocity $V_{f}$, the slope angle $\xi_{j}$ will change by $d \xi$, then the "shooting" method is applied to determine the next values of $a_{j}$ and $V_{f}$, and further computations are accomplished within the outer loop until a minimum of $V_{f}$ or extreme values of $\xi$ will be reached.

As compared with a hypothetical algorithm that would scan the whole interval $\left[\xi_{\min }, \xi_{\max }\right]$ for finding a global minimum of final velocity in two-parametric space, the suggested algorithm detects only a local minimum, so generating a kind of suboptimal solution, but it takes much less runtime of the on-board computer. The same case study as the one examined in the one-parametric guidance has been used in computer simulation of the proposed two-parametric algorithm. The Runge-Kutta time step for integrating the on-board model equations has increased tenfold up to $d t_{m}=0.10 \mathrm{~s}$, and the angle step is set $d \xi=5 \times 10^{-7} \mathrm{rad}$. It should be noted that, for most 
entry trajectories within the attainability domain $\left[x_{t_{f \text { min }}}, x_{t_{f \max }}\right]$, the control profiles $\alpha(x)$ take a characteristic downward stepped from shown in Fig. 6(b), graphs 1 and 2 , where the first part of the entry trajectory is carried out under the negative lift by setting the AoA to the maximum $\alpha_{\max }$ and then, after passing a linear transition stage, the AoA is set to minimum, providing a maximum lift force.

The results of simulation are presented in Fig. 7(a,b). First of all, comparing these data with results obtained in the case of one-parametric guidance (see Fig. 4(b)), it is seen that the marked decrease in the final velocity $V_{f}$ is achieved within the investigated range of coefficient of model discrepancy $k \in[0.5,2.0]$. Unfortunately, there still exist large downrange misses $\Delta x_{f}$, especially disadvantageous for $k>1$ (see graphs CF and CG in Fig. 7(a), which display inability of the proposed two-parametric algorithm to work properly in the case of denser atmosphere.

In order to improve the downrange accuracy, the adaptation algorithm analogous to that described in Section 5 has been applied. The simulation results are shown in Fig. 8. As compared with non-adaptive guidance, one can see considerable improvement in downrange
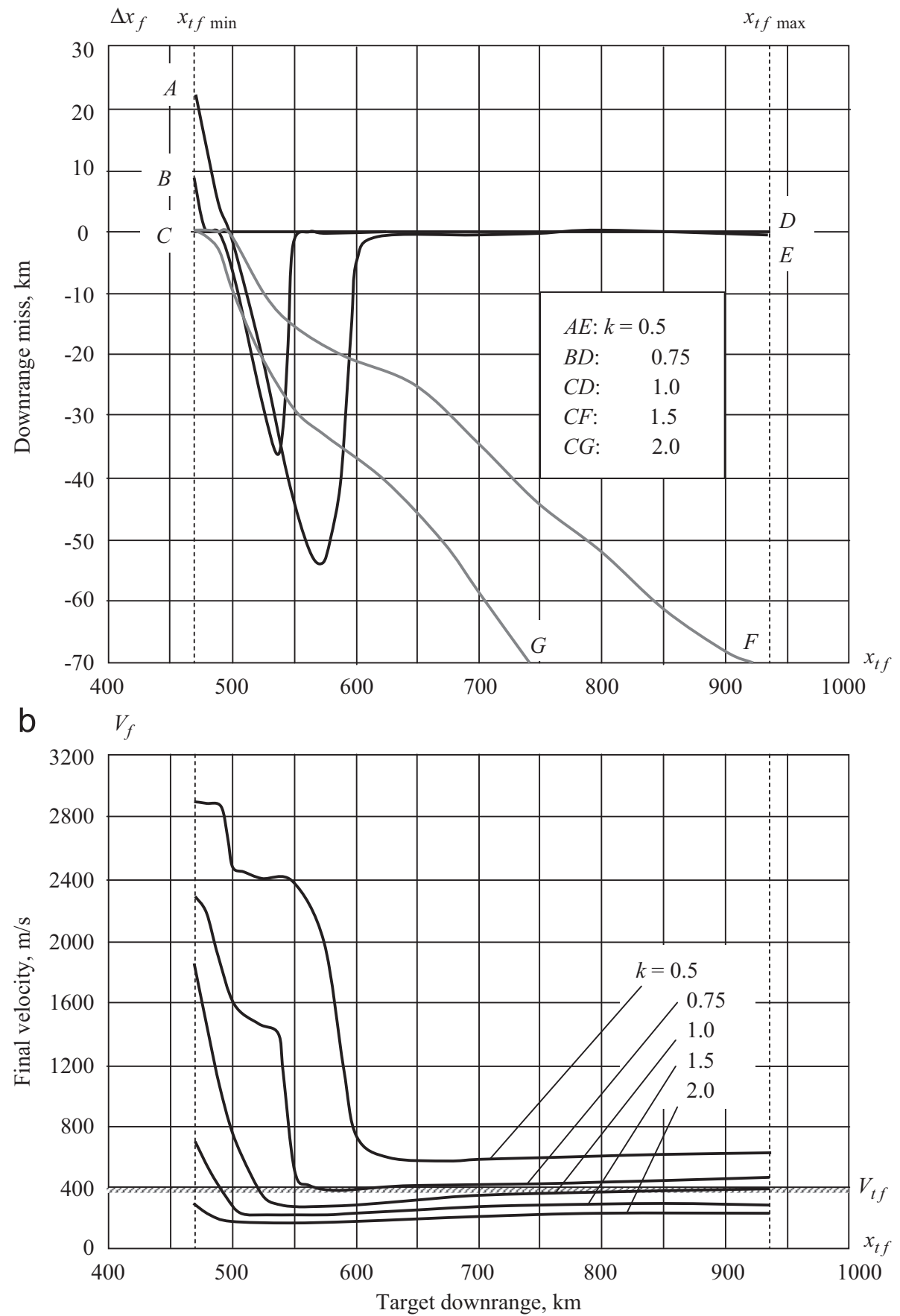

Fig. 7. Two-parametric predictive guidance: dependences of downrange miss $\Delta x_{f}$ (a) and final velocity $V_{f}$ (b) on target downrange $x_{t_{f}}$ and coefficient of model discrepancy $k$. 
a Interval between control corrections $d T=5 \mathrm{sec}$

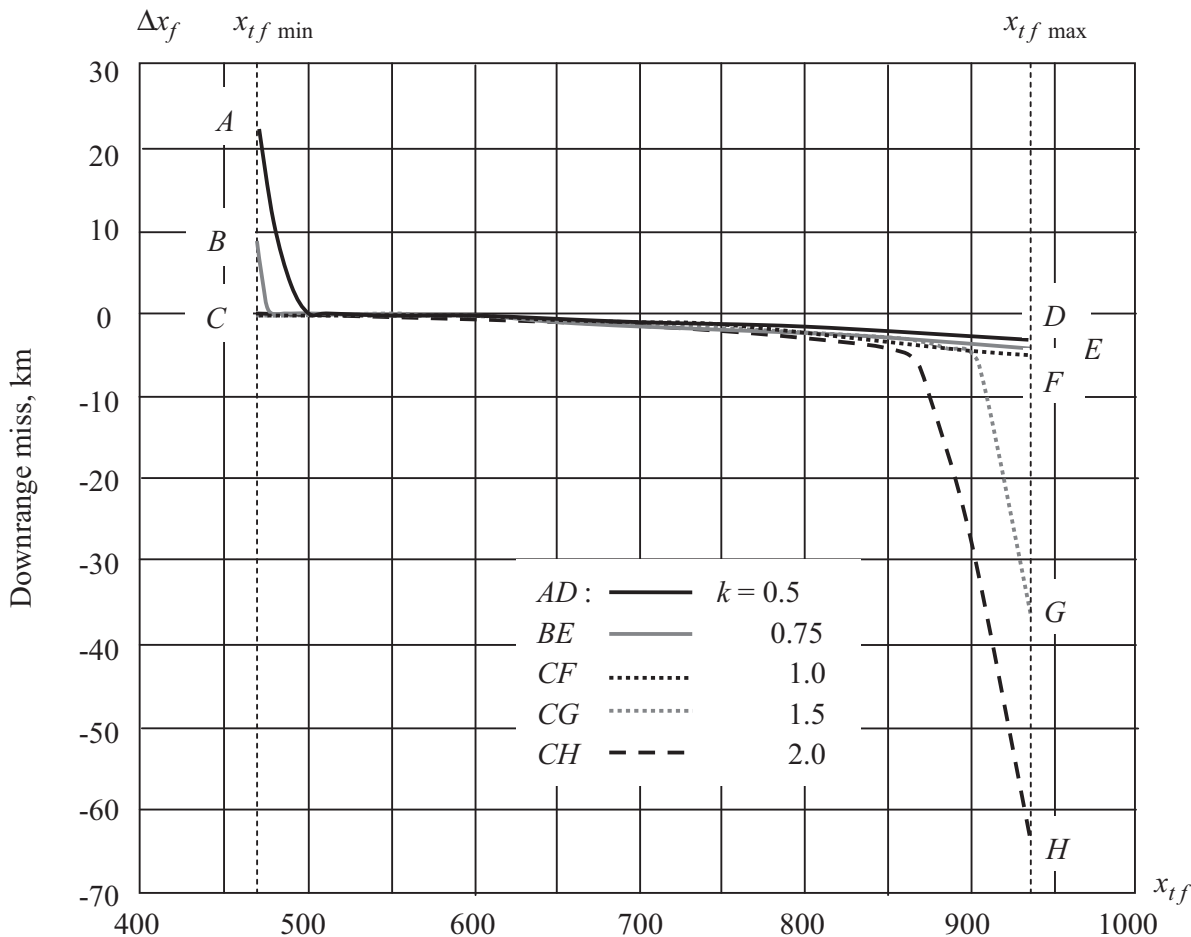

b

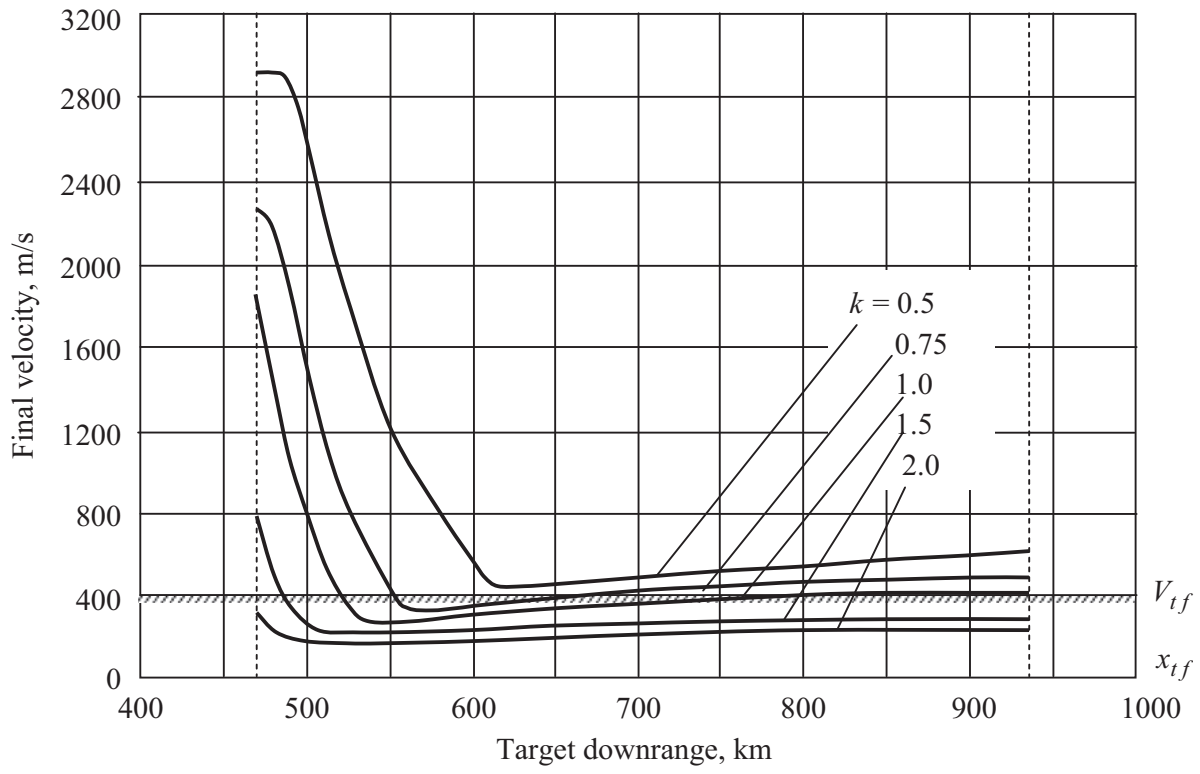

Fig. 8. Two-parametric predictive guidance with model adaptation: dependences of downrange miss $\Delta x_{f}$ (a) and final velocity $V_{f}$ (b) on target downrange $x_{t_{f}}$ and coefficient of model discrepancy $k$.

accuracy for all $k$ belonging to the investigated range. For example, at $k=2$ a broad range (ca. $380 \mathrm{~km}$ long) with relatively small downrange miss $\left|\Delta x_{f}\right|<4 \mathrm{~km}$ has appeared, which consists of $82 \%$ of the nominal attainability domain.

The two-parametric algorithm described above requires increased computational power of on-board computer, so time efficiency of the algorithm should be estimated, at least roughly. For example, let us examine the case of entry guidance without adaptation at the target downrange $x_{t_{f}}=525 \mathrm{~km}$, which may be considered to be one of the worst cases in terms of number of iterations in the outer loop. The first computation of the control profile $\alpha(x)$ at the entry point requires 41 iterations in the outer loop, a maximal number of iterations in the inner loop (path integrations) equals to 22 , and an upper limit to the number of Runge-Kutta integration steps over a path can be estimated for the trajectory with a maximal target downrange $x_{t_{f \max }}=935$ $\mathrm{km}$, i.e., as $t_{f \max } / d t_{m}=445 / 0.1=4450$. Therefore, a total number of integration steps is less than $41224450 \approx 4 \times 10^{6}$. The simulation implemented with a processor AMD Athlon ${ }^{\mathrm{TM}} 64 \times 2$ Dual Core $2.41 \mathrm{GHz}$ shows that the processor time for the given case equals 
to $4.3 \mathrm{~s}$, which is less than chosen interval between control corrections $d T=5.0 \mathrm{~s}$. Introducing the adaptation procedure does not affect the run time appreciably, because the time interval $d T$ for solving an eigenproblem in the adaptation algorithm is much less than the time span $t_{f}$ in predictive guidance algorithms $\left(d T \ll t_{f}\right)$.

\section{Conclusions}

In this paper, a rather challenging problem of applying the predictive methodology and technique to entry guidance of a Mars lander has been considered. The main task of the given research was to find such a guidance algorithm that, being operated in highly changeable and unforeseen environment, could both bring a lander into the predetermined target point and decelerate it to a final velocity small enough for safe parachute deployment. Actually, the research consists of two parts. First, the conventional one-parametric "shooting" guidance technique like those intended for low-lift re-entry vehicles in the Earth atmosphere has been investigated over wide limits of varying both model parameters and target downrange. The results of computer simulation showed that the downrange accuracy proved to be unacceptable under high model discrepancies.

Therefore, aerodynamic and atmospheric uncertainties should be mitigated more radically than it is usually done by repetitive control corrections. A kind of model adaptation algorithm is suggested and examined. Although it suppressed indeed the downrange target errors, the problem of proper decelerating of a lander of the end of entry phase remained urgent and challenging.

A natural way to address this issue is to consider more complex algorithms, e.g., two-parametric predictive algorithms, which are the subject of the second part of the research. Such an algorithm based on a linear control function $\alpha(x)$ has been proposed; its performance being studied on the simulation model. Just as in the oneparametric guidance, the adaptation algorithm has been successfully applied to reduce the downrange miss. The two-parametric algorithm decreases a final velocity $V_{f}$ quite markedly, enhancing the domain of admissible trajectories. For instance, at $k=0.75$ and $x_{t_{f}}=550 \mathrm{~km}$ the final velocity is reduced from 932 to $421 \mathrm{~m} / \mathrm{s}$, and the domain in question of length ca. $110 \mathrm{~km}$ has appeared. The computational burden of the two-parametric algorithm allows to get a solution in real time within several seconds, not exceeding in a worst case the time interval between control corrections $d T=5-7 \mathrm{~s}$.

Therefore, the results obtained in the paper evidently show the feasibility of a predictive guidance approach for the future Mars lander missions. However, the subsequent development should include a comparative performance study of the proposed predictive technique and referencepath tracking guidance, e.g. the Apollo (2nd entry) guidance scheme, as has been done by Kluever [1] for his algorithm of predictive guidance.

\section{References}

[1] C.A. Kluever, Entry guidance performance for Mars precision landing, Journal of Guidance, Control, and Dynamics 31 (2008) 1537-1544.

[2] K.D. Mease, J.A. Leavitt, J. Benito, S. Talole, A. Salama, G. Sohl, M Ivanov, L. Ling, Advanced hypersonic entry guidance for Mars pinpoint landing, in: Proceedings of the NASA Science Technology Conference (NSTC2007), University of Maryland University College, MD, USA, 19-21 June 2007.

[3] N.M. Ivanov, A.I. Martynov, Spacecraft Motion Control in the Atmosphere of Mars, Nauka (Science), Moscow, 1977 in Russian.

[4] J.M. Lafleur, C.J. Cerimele, Mars entry bank profile design for terminal state optimization, in: Proceedings of the AIAA Atmospheric Flight Mechanics Conference and Exhibit, AIAA 2008-6213 Honolulu, HI, USA, 18-21 August 2008.

[5] E. García-Llama, Apollo-derived terminal control for bank-modulated Mars entries with altitude maximization, in: Proceedings of the AIAA Guidance, Navigation and Control Conference and Exhibit AIAA 2008-6819, Honolulu, HI, 18-21 August 2008.

[6] D.W. Way, R.W. Powell, A. Chen, A.D. Steltzner, A.M. San Martin, P.D. Burkhart, G.F. Mendeck, Mars Science Laboratory: entry, descent, and landing system performance, in: Proceedings of the IEEE Aerospace Conference, Big Sky, MT, 3-10 March 2007.

[7] R.D. Braun, R.M. Manning, Mars exploration entry, descent, and landing challenges, Journal of Spacecraft and Rockets 44 (2007) 310-323.

[8] A.M. Korzun, G.F. Dubos, C.K. Iwata, B.A. Stahl, J.J. Quicksall, A concept for the entry, descent, and landing of high-mass payloads at Mars, Acta Astronautica 66 (2010) 1146-1159.

[9] K.-Y. Tu, M.S. Munir, K.D. Mease, D.S. Bayard, Drag-based predictive tracking guidance for Mars precision landing, Journal of Guidance, Control, and Dynamics 23 (2000) 620-628.

[10] G.L. Carman, D.G. Ives, D.K. Geller, Apollo-derived Mars precision landing guidance, in: Proceedings of the AIAA Atmospheric Flight Mechanics Conference, AIAA 1998-4570, Boston, MA, 10-12 August, 1998

[11] G.F. Mendeck, G.L. Carman, Guidance design for Mars Smart Landers using the entry terminal point controller, in: Proceedings of the AIAA Atmospheric Flight Mechanics Conference and Exhibit, AIAA 2002-4502, Monterey, CA, 5-8 August, 2002.

[12] R.W. Powell, Numerical roll reversal predictor-corrector aerocapture and precision landing guidance algorithms for the Mars Surveyor Program 2001 missions, in: Proceedings of the AIAA Atmospheric Flight Mechanics Conference, AIAA 1998-4574, Boston, MA, 10-12 August, 1998.

[13] R.Y. Jits, G.D. Walberg, Blended control, predictor-corrector guidance algorithm: an enabling technology for Mars aerocapture, Acta Astronautica 54 (2004) 385-398.

[14] W.H. Press, S.A. Teukolsky, W.T. Vetterling, B.P. Flannery, Two-point boundary-value problems, Numerical Recipes: the Art of Scientific Computing, third ed., Cambridge University Press, Oxford, 2007 p. 958 (Chapter 18).

[15] G. Hall, J.M. Watt (Eds.), Part 3: Boundary Value Problems, Clarendon Press, Oxford, 1976.

[16] F.J. Regan, S.M. Anandakrishnan, Dynamics of atmospheric re-entry, AIAA Education Series, AIAA, Inc., 1993 Appendix H: Establishment of axis systems and directional cosine matrices.

[17] B.I. Moroz, V.V. Kerzhanovich, V.A. Krasnopolsky, Engineering model of the Martian atmosphere for the Mars-94 project (MA-90), Kosmicheskie Issledovania (Cosmic Research) 29 (1991) 3-84 in Russian.

[18] A.I. Kozynchenko, Fast algorithm for predictive guaranteeing control with application to low-lift re-entry, Journal of Algorithms \& Computational Technology 3 (2009) 289-302.

[19] A.I. Kozynchenko, Predictive guidance algorithms for maximal downrange manoeuvrability with application to low-lift re-entry, Acta Astronautica 64 (2009) 770-777.

[20] A.I. Kozynchenko, Enhancing the manoeuvring capabilities of a lunar landing module using predictive guidance algorithms, Acta Astronautica 67 (2010) 407-417.

[21] N.N. Kalitkin, Numerical Methods, Nauka (Science), Moscow, 1978 p. 280 (in Russian)

[22] A.I. Mourikis, N. Trawny, S.I. Roumeliotis, A.E. Johnson, A. Ansar, L. Matthies, Vision-aided inertial navigation for spacecraft entry, descent, and landing. IEEE Transactions on Robotics 25 (2009) 264-280. 